\title{
In-medium properties of the low-lying strange, charm, and bottom baryons in the quark-meson coupling model
}

\author{
K. Tsushima* \\ Laboratório de Física Teórica e Computacional-LFTC, Universidade Cruzeiro do Sul, \\ 01506-000, São Paulo, SP, Brazil
}

(Received 23 October 2018; published 17 January 2019)

\begin{abstract}
In-medium properties of the low-lying strange, charm, and bottom baryons in symmetric nuclear matter are studied in the quark-meson coupling (QMC) model. Results for the Lorentz-scalar effective masses, mean field potentials felt by the light quarks in the baryons, in-medium bag radii, and the lowest mode bag eigenvalues are presented for those calculated using the updated data. This study completes the in-medium properties of the low-lying baryons in symmetric nuclear matter in the QMC model, for the strange, charm, and bottom baryons which contain one or two strange, one charm, or one bottom quark, as well as at least one light quark. The highlight is the prediction of the bottom baryon Lorentz-scalar effective masses; namely, the Lorentz-scalar effective mass of $\Sigma_{b}$ becomes smaller than that of $\Xi_{b}$ at moderate nuclear matter density, $m_{\Sigma_{b}}^{*}<m_{\Xi_{b}}^{*}$, although in vacuum $m_{\Sigma_{b}}>m_{\Xi_{b}}$. We study further the effects of the repulsive Lorentzvector potentials on the excitation (total) energies of these bottom baryons.
\end{abstract}

DOI: 10.1103/PhysRevD.99.014026

\section{INTRODUCTION}

The study of baryon properties in a nuclear medium, especially for the baryons which contain charm and/or bottom quarks, is very interesting [1-8], due to the emergence of heavy-quark symmetry also in the baryon sector [9-11]. The existence of heavy quarks in hadrons makes it simpler to treat them in many cases; e.g., one can treat them in a nonrelativistic framework with effective potentials such as nonrelativistic QCD [12,13]. In particular, in-medium properties of heavy baryons which contain at least one light $u$ or $d$ quark, can provide us with important information on the dynamical chiral symmetry breaking and the roles of light quarks in partial restoration of chiral symmetry [14-16]. Despite their importance, only a few theoretical studies for the in-medium properties of heavy baryons seem to exist [16-18], probably because of the lack of models and/or methods which are simple enough to handle easily.

To study the in-medium properties of heavy baryons, we rely here on the quark-meson coupling (QMC) model, a quark-based model of nuclear matter, finite nuclei, and hadron properties in a nuclear medium. The model was invented by Guichon [19]. (For other variants of the QMC model, see Ref. [14].) The QMC model has successfully

\footnotetext{
*kazuo.tsushima@gmail.com
}

Published by the American Physical Society under the terms of the Creative Commons Attribution 4.0 International license. Further distribution of this work must maintain attribution to the author(s) and the published article's title, journal citation, and DOI. Funded by SCOAP. been applied for various studies of the properties of finite (hyper)nuclei [20-30], hadron properties in a nuclear medium [31-36], reactions involving nuclear targets [37-45], and neutron star structure [46,47]. Self-consistent exchange of the Lorentz-scalar-isoscalar $\sigma$-, Lorentz-vectorisoscalar $\omega$-, and Lorentz-vector-isovector $\rho$-mean fields, directly coupling to the light quarks $u$ and $d$, is the key feature of the model to be able to achieve the novel saturation properties of nuclear matter with a simple and systematic treatment. All the relevant coupling constants of the $\sigma$ light quark, $\omega$ light quark, and $\rho$ light quark in any hadron are the same as those in the nucleon, those fixed by the nuclear matter saturation properties. The physics behind this simple picture may be supported by the fact that the light-quark condensates reduce or change faster than those of the strange and heavier quarks in finite density as the nuclear density increases $[48,49]$. Or, partial restoration of chiral symmetry in a nuclear medium is mainly driven by the decrease in the magnitude of the light-quark condensates. This is modeled in the QMC model by the fact that the scalar-isoscalar $\sigma$-, vector-isoscalar $\omega$-, and vector-isovector $\rho$-mean fields couple directly only to the light quarks, but not to the strange or heavier quarks.

The present article completes the studies for the lowlying baryon properties in symmetric nuclear matter in the QMC model with some updates. In particular, the highlight is on the bottom baryon Lorentz-scalar effective masses in a nuclear medium. Detailed results are presented explicitly, and many of them have not been presented before $[14,15]$.

We predict that the Lorentz-scalar effective mass of $\Sigma_{b}$ becomes smaller than that of $\Xi_{b}$ at moderate nuclear matter 
density, namely, $m_{\Sigma_{b}}^{*}<m_{\Xi_{b}}^{*}$, although $m_{\Sigma_{b}}>m_{\Xi_{b}}$ in vacuum. We study further the effects of the repulsive Lorentz-vector potentials on the excitation (total) energies of these bottom baryons, by considering two different possibilities for the vector potentials: one is extracted by the $\Lambda$ and $\Sigma$ hypernuclear experimental observation, the one which includes effective Pauli potentials based on the Pauli principle at the quark level, and the other is the vector potentials that are predicted by the QMC model without the effective Pauli potentials.

\section{FINITE (HYPER)NUCLEUS IN THE QMC MODEL}

In order to make this article self-contained, we briefly review the QMC model following Refs. [14,15] with minor improvements for better understanding.

Although the Hartree-Fock treatment is possible within the QMC model [50], the main features of the results, especially the density dependence of the total energy per nucleon (nuclear matter energy density), is nearly identical to that of the Hartree approximation. Then, it is sufficient to rely on the Hartree approximation in this study. (See Ref. [46] for a detailed study made for the neutron star structure based on the QMC model with the Hartree-Fock treatment.)

Before discussing the heavy baryon properties in symmetric nuclear matter, we start with the case of a finite (hyper)nucleus. Using the Born-Oppenheimer approximation, a relativistic Lagrangian density which gives the same mean-field equations of motion for a nucleus or a hypernucleus, may be given in the QMC model $[14,15,25]$ by Eqs. (1)-(3) below, where the quasiparticles moving in single-particle orbits are threequark clusters with the quantum numbers of a nucleon, strange, charm, or bottom hyperon when expanded to the same order in velocity $[20,21,25,28,30,36]$ :

$$
\begin{gathered}
\mathcal{L}_{Q M C}=\mathcal{L}_{\mathrm{QMC}}^{N}+\mathcal{L}_{\mathrm{QMC}}^{Y}, \\
\mathcal{L}_{\mathrm{QMC}}^{N} \equiv \bar{\psi}_{N}(\vec{r})\left[i \gamma \cdot \partial-m_{N}^{*}(\sigma)-\left(g_{\omega} \omega(\vec{r})+g_{\rho} \frac{\tau_{3}^{N}}{2} b(\vec{r})+\frac{e}{2}\left(1+\tau_{3}^{N}\right) A(\vec{r})\right) \gamma_{0}\right] \psi_{N}(\vec{r}) \\
-\frac{1}{2}\left[(\nabla \sigma(\vec{r}))^{2}+m_{\sigma}^{2} \sigma(\vec{r})^{2}\right]+\frac{1}{2}\left[(\nabla \omega(\vec{r}))^{2}+m_{\omega}^{2} \omega(\vec{r})^{2}\right]+\frac{1}{2}\left[(\nabla b(\vec{r}))^{2}+m_{\rho}^{2} b(\vec{r})^{2}\right]+\frac{1}{2}(\nabla A(\vec{r}))^{2}, \\
\mathcal{L}_{\mathrm{QMC}}^{Y} \equiv \bar{\psi}_{Y}(\vec{r})\left[i \gamma \cdot \partial-m_{Y}^{*}(\sigma)-\left(g_{\omega}^{Y} \omega(\vec{r})+g_{\rho}^{Y} I_{3}^{Y} b(\vec{r})+e Q_{Y} A(\vec{r})\right) \gamma_{0}\right] \psi_{Y}(\vec{r}), \\
\left(Y=\Lambda, \Sigma^{0, \pm}, \Xi^{0,-}, \Lambda_{c}^{+}, \Sigma_{c}^{0,+,++}, \Xi_{c}^{0,+}, \Lambda_{b}, \Sigma_{b}^{0, \pm}, \Xi_{b}^{0,-}\right),
\end{gathered}
$$

where, for a normal nucleus, $\mathcal{L}_{\mathrm{QMC}}^{Y}$ in Eq. (1), namely Eq. (3) is not needed, but for the following study we do need this. In the above, $\psi_{N}(\vec{r})$ and $\psi_{Y}(\vec{r})$ are, respectively, the nucleon and hyperon (strange, charm, or bottom baryon) fields. The mean-meson fields represented by, $\sigma, \omega$, and $b$, which directly couple to the light quarks self-consistently, are the Lorentz-scalar-isoscalar, Lorentzvector-isoscalar, and third component of the Lorentzvector-isovector fields, respectively, while $A$ stands for the Coulomb field.

In an approximation where the $\sigma$-, $\omega$-, and $\rho$-mean fields couple only to the $u$ and $d$ light quarks, the coupling constants for the hyperon appearing in Eq. (3) are obtained/ identified as $g_{\omega}^{Y}=\left(n_{q} / 3\right) g_{\omega}$, and $g_{\rho}^{Y} \equiv g_{\rho}=g_{\rho}^{q}$, with $n_{q}$ being the total number of valence light quarks in the hyperon $Y$, where $g_{\omega}$ and $g_{\rho}$ are the $\omega-N$ and $\rho-N$ coupling constants. $I_{3}^{Y}$ and $Q_{Y}$ are the third component of the hyperon isospin operator and its electric charge in units of the proton charge, $e$, respectively.

As mentioned already, the approximation adopted in the QMC model, that the meson fields couple only to the light quarks, reflects the fact that the magnitudes of the lightquark condensates decrease faster as increasing the nuclear density than those of the strange and heavy flavor quarks. This is associated with the partial restoration of chiral symmetry in a nuclear medium (dynamical symmetry breaking and its partial restoration). The dynamical symmetry breaking and its restoration can provide us with important information on the origin of the (dynamical) masses of the hadrons which we observe in our Universe.

The field dependent $\sigma-N$ and $\sigma-Y$ coupling strengths, respectively, for the nucleon $N$ and hyperon $Y, g_{\sigma}(\sigma) \equiv$ $g_{\sigma}^{N}(\sigma)$ and $g_{\sigma}^{Y}(\sigma)$ appearing in Eqs. (2) and (3), are defined by

$$
\begin{aligned}
m_{N}^{*}(\sigma) \equiv & m_{N}-g_{\sigma}(\sigma) \sigma(\vec{r}) \\
m_{Y}^{*}(\sigma) \equiv & m_{Y}-g_{\sigma}^{Y}(\sigma) \sigma(\vec{r}) \\
& \quad\left(Y=\Lambda, \Sigma, \Xi, \Lambda_{c}, \Sigma_{c}, \Xi_{c}, \Lambda_{b}, \Sigma_{b}, \Xi_{b}\right),
\end{aligned}
$$

where $m_{N}\left(m_{Y}\right)$ is the free nucleon (hyperon) mass. Note that the dependence of these coupling strengths on the 
applied scalar field $(\sigma)$ must be calculated self-consistently within the quark model $[19,20,25,28,29,36]$. Hence, unlike quantum hadrodynamics (QHD) [51,52], even though $g_{\sigma}^{Y}(\sigma) / g_{\sigma}(\sigma)$ may be $2 / 3$ or $1 / 3$ depending on the number of light quarks $n_{q}$ in the hyperon in free space, $\sigma=0$ (even this is true only when their bag radii in free space are exactly the same in the standard QMC model with the MIT bag), this will not necessarily be the case in a nuclear medium.

The Lagrangian density Eq. (1) leads [or (2) and (3) lead] to a set of equations of motion for the finite (hyper)nuclear system:

$$
\begin{gathered}
{\left[i \gamma \cdot \partial-m_{N}^{*}(\sigma)-\left(g_{\omega} \omega(\vec{r})+g_{\rho} \frac{\tau_{3}^{N}}{2} b(\vec{r})\right.\right.} \\
\left.\left.+\frac{e}{2}\left(1+\tau_{3}^{N}\right) A(\vec{r})\right) \gamma_{0}\right] \psi_{N}(\vec{r})=0 \\
{\left[i \gamma \cdot \partial-m_{Y}^{*}(\sigma)-\left(g_{\omega}^{Y} \omega(\vec{r})+g_{\rho}^{Y} I_{3}^{Y} b(\vec{r})\right.\right.} \\
\left.\left.+e Q_{Y} A(\vec{r})\right) \gamma_{0}\right] \psi_{Y}(\vec{r})=0 \\
\left(-\nabla_{r}^{2}+m_{\sigma}^{2}\right) \sigma(\vec{r})=-\left[\frac{\partial m_{N}^{*}(\sigma)}{\partial \sigma}\right] \rho_{s}(\vec{r})-\left[\frac{\partial m_{Y}^{*}(\sigma)}{\partial \sigma}\right] \rho_{s}^{Y}(\vec{r}), \\
\equiv g_{\sigma} C_{N}(\sigma) \rho_{s}(\vec{r})+g_{\sigma}^{Y} C_{Y}(\sigma) \rho_{s}^{Y}(\vec{r}) \\
\left(-\nabla_{r}^{2}+m_{\omega}^{2}\right) \omega(\vec{r})=g_{\omega} \rho_{B}(\vec{r})+g_{\omega}^{Y} \rho_{B}^{Y}(\vec{r}) \\
\left(-\nabla_{r}^{2}+m_{\rho}^{2}\right) b(\vec{r})=\frac{g_{\rho}}{2} \rho_{3}(\vec{r})+g_{\rho}^{Y} I_{3}^{Y} \rho_{B}^{Y}(\vec{r}) \\
\left(-\nabla_{r}^{2}\right) A(\vec{r})=e \rho_{p}(\vec{r})+e Q_{Y} \rho_{B}^{Y}(\vec{r}),
\end{gathered}
$$

where $\rho_{s}(\vec{r}) \quad\left[\rho_{s}^{Y}(\vec{r})\right], \quad \rho_{B}(\vec{r})=\rho_{p}(\vec{r})+\rho_{n}(\vec{r}) \quad\left[\rho_{B}^{Y}(\vec{r})\right]$, $\rho_{3}(\vec{r})=\rho_{p}(\vec{r})-\rho_{n}(\vec{r}), \rho_{p}(\vec{r})$, and $\rho_{n}(\vec{r})$ are the nucleon (hyperon) scalar; nucleon (hyperon) baryon; and third component of the isovector, proton, and neutron densities at the position $\vec{r}$ in the (hyper)nucleus, respectively. On the right-hand side of Eq. (8), $-\left[\partial m_{N}^{*}(\sigma) / \partial \sigma\right] \equiv g_{\sigma} C_{N}(\sigma)$ and $-\left[\partial m_{Y}^{*}(\sigma) / \partial \sigma\right] \equiv g_{\sigma}^{Y} C_{Y}(\sigma)$, where $g_{\sigma} \equiv g_{\sigma}(\sigma=0)$ and $g_{\sigma}^{Y} \equiv g_{\sigma}^{Y}(\sigma=0)$ hereafter in this article, are the key ingredients of the QMC model. Note that, when there is $\sigma$ dependence, they will be explicitly written by $g_{\sigma}(\sigma)$ and $g_{\sigma}^{Y}(\sigma)$ to avoid confusion. At the hadronic level, the entire information of the quark dynamics is condensed in the effective couplings $C_{N, Y}(\sigma)$ of Eq. (8), which characterize the features of the QMC model, namely, scalar polarizability. Furthermore, when $C_{N, Y}(\sigma)=1$, which corresponds to a structureless nucleon or hyperon, the equations of motion given by Eqs. (6)-(11) can be identified with those derived from naive QHD [51,52].

We note that, for the Dirac equation Eq. (7) for the hyperon $Y$, we include the effects due to the Pauli blocking at the quark level by adding repulsive potentials based on the study made for the strange hyperons $\Lambda, \Sigma$, and $\Xi$. The net, repulsive "Pauli potentials," which may be interpreted as also including the $\Lambda N-\Sigma N$ channel coupling effect, were extracted by the fit to the $\Lambda$ and $\Sigma$ hypernuclei taking into account the $\Sigma N-\Lambda N$ channel coupling [25]. Of course, the effects of the channel coupling are expected to be smaller for the corresponding charm and bottom baryons, since the corresponding mass differences for these cases are larger than that for the $\Lambda$ and $\Sigma$ hyperons. Thus, for the interesting case of the $\Sigma_{b}-\Xi_{b}$ baryon system focused on later, we study two possibilities of the vector potentials, with and without including the effective Pauli potentials. The modified Dirac equation for $Y=\Lambda, \Sigma, \Xi$, $\Lambda_{c, b}, \Sigma_{c, b}$, and $\Xi_{c, b}$ is

$$
\begin{aligned}
& {\left[i \gamma \cdot \partial-M_{Y}(\sigma)-\left(\lambda_{Y} \rho_{B}(\vec{r})+g_{\omega}^{Y} \omega(\vec{r})+g_{\rho} I_{3}^{Y} b(\vec{r})\right.\right.} \\
& \left.\left.\quad+e Q_{Y} A(\vec{r})\right) \gamma_{0}\right] \psi_{Y}(\vec{r})=0,
\end{aligned}
$$

where $\lambda_{Y} \rho_{B}(\vec{r})$ is the effective Pauli potential for the hyperon $Y$, with $\rho_{B}(\vec{r})$ being the baryon density at the position $\vec{r}$ in the corresponding hypernucleus. The values of $\lambda_{Y}$ for $Y=\left(\Lambda, \Lambda_{c, b}\right)$ and $\left(\Sigma, \Sigma_{c, b}\right)$ are, respectively, $60.25 \mathrm{MeV}(\mathrm{fm})^{3}$ and $110.6 \mathrm{MeV}(\mathrm{fm})^{3}$, while for $Y=\Xi$ and $\Xi_{c, b}, \lambda_{Y}$ is $(1 / 2) \times 60.25 \mathrm{MeV}(\mathrm{fm})^{3}$ based on the valence light-quark number. For the details of the effective Pauli potentials at the quark level, see Ref. [25].

The effective masses of the nucleon $N\left(m_{N}^{*}\right)$ and hyperon $Y\left(m_{Y}^{*}\right)$ are calculated later by Eq. (25) (by replacing $h \rightarrow N$ and $h \rightarrow Y$, respectively, there). The explicit expressions for $C_{N, Y}(\sigma) \equiv S_{N, Y}(\sigma) / S_{N, Y}(\sigma=0)\left[S_{N, Y}(\sigma)\right.$ to be defined next] and the effective masses $m_{N, Y}^{*}$ are related by

$$
\begin{aligned}
\frac{\partial m_{N, Y}^{*}(\sigma)}{\partial \sigma} & =-n_{q} g_{\sigma}^{q} \int_{\mathrm{bag}} d^{3} y \bar{\psi}_{q}(\vec{y}) \psi_{q}(\vec{y}) \\
& \equiv-n_{q} g_{\sigma}^{q} S_{N, Y}(\sigma)=-\left[n_{q} g_{\sigma}^{q} S_{N, Y}(\sigma=0)\right] C_{N, Y}(\sigma) \\
& =-\frac{\partial}{\partial \sigma}\left[g_{\sigma}^{N, Y}(\sigma) \sigma\right],
\end{aligned}
$$

where $g_{\sigma}^{q}$ is the light-quark- $\sigma$ coupling constant and $\psi_{q}$ is the light-quark ground state wave function in the nucleon $N$ or hyperon $Y$ immersed in a nuclear medium. By the above relation, we define the $\sigma-N$ and $\sigma-Y$ coupling constants,

$$
g_{\sigma}^{N, Y} \equiv n_{q} g_{\sigma}^{q} S_{N, Y}(\sigma=0)
$$

where $g_{\sigma}^{N} \equiv g_{\sigma}=g_{\sigma}(\sigma=0)$ appeared already. Note that, as in the case of $C_{N, Y}(\sigma)$, the values of $S_{N}(\sigma=0)$ and $S_{Y}(\sigma=0)$ are different, because the light-quark wave functions in the nucleon $N$ and hyperon $Y$ are different in vacuum as well as in medium; that is, the bag radii of the $N$ and $Y$ are different in both vacuum and medium.

The parameters appearing at the nucleon, hyperon, and meson Lagrangian level used for the study of infinite 
nuclear matter and finite nuclei $[20,21]$ are $m_{\omega}=783 \mathrm{MeV}$, $m_{\rho}=770 \mathrm{MeV}, m_{\sigma}=550 \mathrm{MeV}$, and $e^{2} / 4 \pi=1 / 137.036$. (See Ref. [21] for a discussion on the parameter fixing in the QMC model, especially in treating finite nuclei.)

\section{BARYON PROPERTIES IN SYMMETRIC NUCLEAR MATTER}

We consider the rest frame of infinitely large, symmetric nuclear matter, a spin and isospin saturated system with only strong interaction (the Coulomb force is dropped as usual). One first keeps only $\mathcal{L}_{\mathrm{QMC}}^{N}$ in Eq. (1), or correspondingly drops all the quantities with the superscripts and subscripts $Y$, and sets the Coulomb field $A(\vec{r})=0$ in Eqs. (6)-(11). Next one sets all the terms with any derivatives of the fields to be zero. Then, within the Hartree mean-field approximation, the nuclear (baryon) $\rho_{B}$ and scalar $\rho_{s}$ densities are, respectively, given by

$$
\begin{gathered}
\rho_{B}=\frac{4}{(2 \pi)^{3}} \int d^{3} k \theta\left(k_{F}-|\vec{k}|\right)=\frac{2 k_{F}^{3}}{3 \pi^{2}}, \\
\rho_{s}=\frac{4}{(2 \pi)^{3}} \int d^{3} k \theta\left(k_{F}-|\vec{k}|\right) \frac{m_{N}^{*}(\sigma)}{\sqrt{m_{N}^{* 2}(\sigma)+\vec{k}^{2}}} .
\end{gathered}
$$

Here, $m_{N}^{*}(\sigma)$ is the value (constant) of the Lorentz-scalar effective nucleon mass at a given nuclear (baryon) density [see also Eq. (4)] and $k_{F}$ the Fermi momentum. In the standard QMC model [19], the MIT bag model is used for describing nucleons and hyperons (hadrons). The use of this quark model is an essential ingredient for the QMC model, namely, the use of the relativistic, confined quarks.

The Dirac equations for the quarks and antiquarks in nuclear matter, in a bag of a hadron, $h(q=u$ or $d$ and $Q=s, c$, or $b$ hereafter), neglecting the Coulomb force, are given by $[x=(t, \vec{x})$ and for $|\vec{x}| \leq$ bag radius] [32,34-37]

$$
\left[i \gamma \cdot \partial_{x}-\left(m_{q}-V_{\sigma}^{q}\right) \mp \gamma^{0}\left(V_{\omega}^{q}+\frac{1}{2} V_{\rho}^{q}\right)\right]\left(\begin{array}{l}
\psi_{u}(x) \\
\psi_{\bar{u}}(x)
\end{array}\right)=0,
$$

$$
\left[i \gamma \cdot \partial_{x}-\left(m_{q}-V_{\sigma}^{q}\right) \mp \gamma^{0}\left(V_{\omega}^{q}-\frac{1}{2} V_{\rho}^{q}\right)\right]\left(\begin{array}{l}
\psi_{d}(x) \\
\psi_{\bar{d}}(x)
\end{array}\right)=0,
$$

$$
\left[i \gamma \cdot \partial_{x}-m_{Q}\right] \psi_{Q}(x)=0, \quad\left[i \gamma \cdot \partial_{x}-m_{Q}\right] \psi_{\bar{Q}}(x)=0,
$$

where the (constant) mean fields for a bag in nuclear matter are defined by $V_{\sigma}^{q} \equiv g_{\sigma}^{q} \sigma, \quad V_{\omega}^{q} \equiv g_{\omega}^{q} \omega$, and $V_{\rho}^{q} \equiv g_{\rho}^{q} b$, with $g_{\sigma}^{q}, g_{\omega}^{q}$, and $g_{\rho}^{q}$ being the corresponding quarkmeson coupling constants. We assume SU(2) symmetry, $m_{u, \bar{u}}=m_{d, \bar{d}} \equiv m_{q, \bar{q}}$. The corresponding Lorentz-scalar effective quark masses are defined by $m_{u, \bar{u}}^{*}=m_{d, \bar{d}}^{*}=$ $m_{q, \bar{q}}^{*} \equiv m_{q, \bar{q}}-V_{\sigma}^{q}$. Since the $\rho$-meson mean field becomes zero, $V_{\rho}^{q}=0$ in Eqs. (17) and (18) in symmetric nuclear matter in the Hartree approximation, we will ignore it. [This is not true in a finite nucleus with equal and more than two protons even with equal numbers of protons and neutrons, since the Coulomb interactions among the protons induce an asymmetry between the proton and neutron density distributions to give $\rho_{3}(\vec{r})=\rho_{p}(\vec{r})-\rho_{n}(\vec{r}) \neq 0$.]

The same meson-mean fields $\sigma$ and $\omega$ for the quarks in Eqs. (17) and (18) satisfy self-consistently the following equations at the nucleon level [together with the Lorentzscalar effective nucleon mass $m_{N}^{*}(\sigma)$ of Eq. (4) to be calculated by Eq. (25)]:

$$
\begin{gathered}
\omega=\frac{g_{\omega}}{m_{\omega}^{2}} \rho_{B}, \\
\sigma=\frac{g_{\sigma}}{m_{\sigma}^{2}} C_{N}(\sigma) \frac{4}{(2 \pi)^{3}} \int d^{3} k \theta\left(k_{F}-|\vec{k}|\right) \frac{m_{N}^{*}(\sigma)}{\sqrt{m_{N}^{* 2}(\sigma)+\vec{k}^{2}}} \\
=\frac{g_{\sigma}}{m_{\sigma}^{2}} C_{N}(\sigma) \rho_{s},
\end{gathered}
$$

where

$$
C_{N}(\sigma) \equiv \frac{-1}{g_{\sigma}(\sigma=0)}\left[\partial m_{N}^{*}(\sigma) / \partial \sigma\right]
$$

Because of the underlying quark structure of the nucleon used to calculate $m_{N}^{*}(\sigma)$ in a nuclear medium, $C_{N}(\sigma)$ decreases as $\sigma$ increases, whereas in the usual pointlike nucleon-based models it is constant, $C_{N}(\sigma)=1$. As will be discussed later, it can be parametrized in the QMC model as $C_{N}(\sigma)=1-a_{N} \times\left(g_{\sigma} \sigma\right)\left(a_{N}>0\right)$. It is this variation of $C_{N}(\sigma)$ [or equivalently the dependence of the scalar coupling on density, or $\left.\sigma, g_{\sigma}(\sigma)\right]$ that yields a novel saturation mechanism for nuclear matter in the QMC model and contains the important dynamics which originates from the quark structure of the nucleons and hadrons. It is the variation of this $C_{N}(\sigma)$, which yields three-body or density dependent effective forces, that has been demonstrated by constructing an equivalent energy density functional [24,53]. As a consequence of the derived, nonlinear couplings of the meson fields in the Lagrangian density at the nucleon (hyperon) and meson level, the standard QMC model yields the nuclear incompressibility of $K \simeq$ $280 \mathrm{MeV}$ with $m_{q}=5 \mathrm{MeV}$. This is in contrast to a naive version of QHD [51,52] (the pointlike nucleon model of nuclear matter), which results in the much larger value, $K \simeq 500 \mathrm{MeV}$; the empirically extracted value falls in the range $K=200-300 \mathrm{MeV}$. (See Ref. [54] for an extensive analysis of this issue.) 
TABLE I. Current quark mass values (inputs), quark-meson coupling constants, and the bag pressure $B_{p}$. Note that the $m_{c}$ value is updated from Refs. [14,15] based on the data [55].

\begin{tabular}{lrll}
\hline \hline$m_{u, d}$ & $5 \mathrm{MeV}$ & $g_{\sigma}^{q}$ & 5.69 \\
$m_{s}$ & $250 \mathrm{MeV}$ & $g_{\omega}^{q}$ & 2.72 \\
$m_{c}$ & $1270 \mathrm{MeV}$ & $g_{\rho}^{q}$ & 9.33 \\
$m_{b}$ & $4200 \mathrm{MeV}$ & $B_{p}^{1 / 4}$ & $170 \mathrm{MeV}$ \\
\hline \hline
\end{tabular}

Once the self-consistency equation for the $\sigma$ field Eq. (21) is solved, one can evaluate the total energy per nucleon:

$$
\begin{aligned}
E^{\mathrm{tot}} / A= & \frac{4}{(2 \pi)^{3} \rho_{B}} \int d^{3} k \theta\left(k_{F}-|\vec{k}|\right) \sqrt{m_{N}^{* 2}(\sigma)+\vec{k}^{2}} \\
& +\frac{m_{\sigma}^{2} \sigma^{2}}{2 \rho_{B}}+\frac{g_{\omega}^{2} \rho_{B}}{2 m_{\omega}^{2}} .
\end{aligned}
$$

We then determine the coupling constants $g_{\sigma}$ and $g_{\omega}$ at the nucleon level [see also Eq. (14)], by the fit to the binding energy of $15.7 \mathrm{MeV}$ at the saturation density $\rho_{0}=$ $0.15 \mathrm{fm}^{-3}\left(k_{F}^{0}=1.305 \mathrm{fm}^{-1}\right)$ for symmetric nuclear matter, as well as $g_{\rho}$ to the symmetry energy of $35 \mathrm{MeV}$. The determined quark-meson coupling constants and the current quark mass values are listed in Table I. The coupling constants at the nucleon level are $g_{\sigma}^{2} / 4 \pi=3.12, g_{\omega}^{2} / 4 \pi=$ 5.31, and $g_{\rho}^{2} / 4 \pi=6.93$. [See Eq. (14) for $g_{\sigma}=g_{\sigma}^{N}$.]

We show in Fig. 1 the density dependence of the total energy per nucleon $E^{\text {tot }} / A-m_{N}$ (left panel) and the Lorentz-scalar effective quark mass $m_{q}^{*}$ and the vector $\left(V_{\omega}^{q}\right)$ and scalar $\left(-V_{\sigma}^{q}\right)$ potentials felt by the light quarks (right panel) calculated using the quark-meson coupling constants determined.

In the following, let us consider the situation that a hadron $h$ (or a hyperon $Y$ ) is immersed in nuclear matter. The normalized, static solution for the ground state quarks

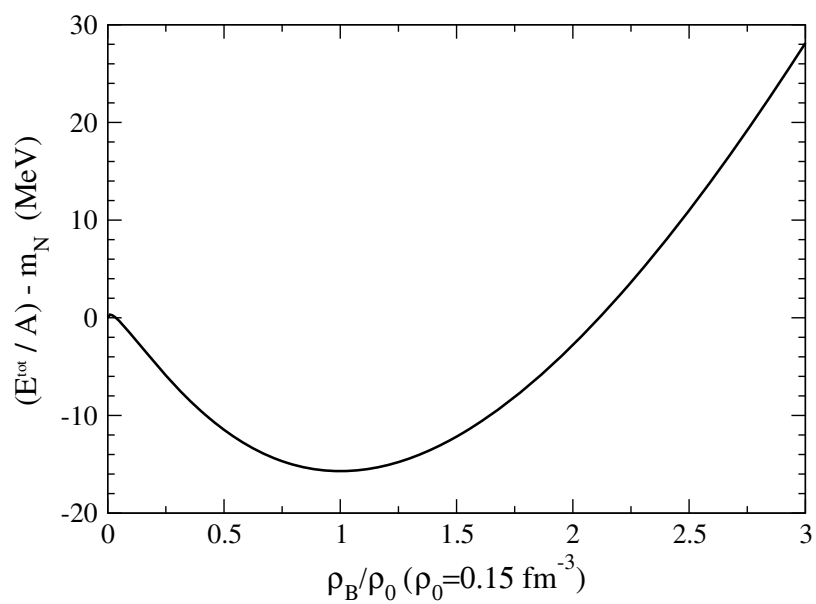

or antiquarks with flavor $f$ in the hadron $h$ may be written $\psi_{f}(x)=N_{f} \exp ^{-i \epsilon_{f} t / R_{h}^{*}} \psi_{f}(\vec{r})$, where $N_{f}$ and $\psi_{f}(\vec{r})$ are the normalization factor and corresponding spin and spatial part of the wave function. The bag radius in a medium for the hadron $h$, denoted by $R_{h}^{*}$, is determined through the stability condition for the mass of the hadron against the variation of the bag radius $[19,26]$ [see Eq. (25)]. The eigenenergies in units of $1 / R_{h}^{*}$ are given by

$$
\begin{aligned}
\left(\begin{array}{c}
\epsilon_{u} \\
\epsilon_{\bar{u}}
\end{array}\right) & =\Omega_{q}^{*} \pm R_{h}^{*}\left(V_{\omega}^{q}+\frac{1}{2} V_{\rho}^{q}\right), \\
\left(\begin{array}{c}
\epsilon_{d} \\
\epsilon_{\bar{d}}
\end{array}\right) & =\Omega_{q}^{*} \pm R_{h}^{*}\left(V_{\omega}^{q}-\frac{1}{2} V_{\rho}^{q}\right), \\
\epsilon_{Q} & =\epsilon_{\bar{Q}}=\Omega_{Q} .
\end{aligned}
$$

The hadron mass in a nuclear medium, $m_{h}^{*}$ (free mass is denoted by $m_{h}$ ), is calculated by

$m_{h}^{*}=\sum_{j=q, \bar{q}, Q, \bar{Q}} \frac{n_{j} \Omega_{j}^{*}-z_{h}}{R_{h}^{*}}+\frac{4}{3} \pi R_{h}^{* 3} B_{p},\left.\quad \frac{\partial m_{h}^{*}}{\partial R_{h}}\right|_{R_{h}=R_{h}^{*}}=0$,

where $\Omega_{q}^{*}=\Omega_{\bar{q}}^{*}=\left[x_{q}^{2}+\left(R_{h}^{*} m_{q}^{*}\right)^{2}\right]^{1 / 2}(q=u, d)$, with $m_{q}^{*}=$ $m_{q}-g_{\sigma}^{q} \sigma=m_{q}-V_{\sigma}^{q}, \quad \Omega_{Q}^{*}=\Omega_{\bar{Q}}^{*}=\left[x_{Q}^{2}+\left(R_{h}^{*} m_{Q}\right)^{2}\right]^{1 / 2}$ $(Q=s, c, b)$, and $x_{q, Q}$ are the lowest mode bag eigenvalues. $B_{p}$ is the bag pressure (constant), $n_{q}\left(n_{\bar{q}}\right)$ and $n_{Q}\left(n_{\bar{Q}}\right)$ are the lowest mode valence quark (antiquark) numbers for the quark flavors $q$ and $Q$ in the hadron $h$, respectively, while $z_{h}$ parametrizes the sum of the center-of-mass and gluon fluctuation effects, which are assumed to be independent of density [20]. The bag pressure $B_{p}=$ $(170 \mathrm{MeV})^{4}$ (density independent) is determined by the free nucleon mass $m_{N}=939 \mathrm{MeV}$ with the bag radius in vacuum $R_{N}=0.8 \mathrm{fm}$ and $m_{q}=5 \mathrm{MeV}$ as inputs, which

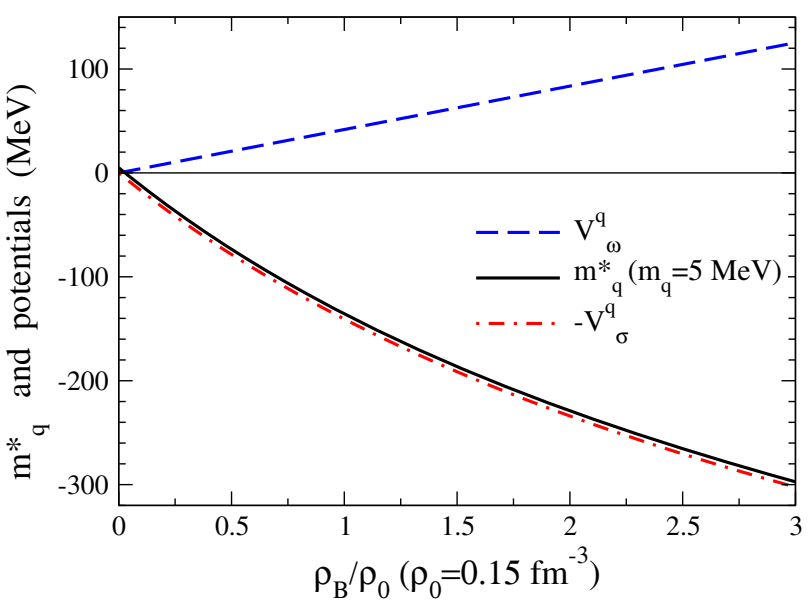

FIG. 1. Total energy per nucleon $E^{\text {tot }} / A-m_{N}$ (left panel), and the light-quark Lorentz-scalar effective mass $m_{q}^{*}$, vector potential $\left(V_{\omega}^{q}\right)$, and scalar potential $\left(-V_{\sigma}^{q}\right)$ felt by the light quarks. 
are considered to be standard values in the QMC model [14]. (See also Table I.) Concerning the Lorentz-scalar effective mass $m_{q}^{*}$ in a nuclear medium, it reflects nothing but the strength of the attractive scalar potential as in Eqs. (17) and (18), and thus the naive interpretation of the mass for a (physical) particle, which is positive, should not be applied. The model parameters are determined to reproduce the corresponding masses in free space. The quark-meson coupling constants, $g_{\sigma}^{q}, g_{\omega}^{q}$, and $g_{\rho}^{q}$, have already been determined by the nuclear matter saturation properties. Exactly the same coupling constants, $g_{\sigma}^{q}, g_{\omega}^{q}$, and $g_{\rho}^{q}$, will be used for the light quarks in all the hadrons as in the nucleon. These values are fixed and will not be changed depending on the hadrons.

In Table II we present the inputs; vacuum masses of baryons $B, m_{B}$; the parameters $z_{B}$; the calculated lowest mode bag eigenvalues $\left(x_{1}, x_{2}, x_{3}\right)$ of the corresponding valence quarks $\left(q_{1}, q_{2}, q_{3}\right)$ in the baryon $B$; and the bag radii calculated in vacuum $R_{B}$, as well as the corresponding quantities at $\rho_{0}=0.15 \mathrm{fm}^{-3}$, namely, the Lorentz-scalar effective masses $m_{B}^{*}$, in-medium bag radii $R_{B}^{*}$, and the lowest mode bag eigenvalues, $\left(x_{1}^{*}, x_{2}^{*}, x_{3}^{*}\right)$. Note that in the QMC model, the $\Omega(s s s), \Omega_{c}(s s c)$, and $\Omega_{b}(s s b)$ properties are not modified in the medium.

One can notice a few things easily in Table II: (i) the parameter $z_{B}$ decreases as the vacuum mass of the baryon increases; (ii) the in-medium bag radius $R_{B}^{*}$ of the baryon $B$ at $\rho_{0}$ decreases and becomes smaller than the corresponding vacuum value, $R_{B}$, and the decreasing ratio becomes smaller as the vacuum baryon mass value increases; and (iii) the lowest mode bag eigenvalues decrease at $\rho_{0}$, and the decreasing magnitude is larger for the light quarks, but tiny for the heavier quarks. Note that the bag radius is not the physical observable, and one must calculate the baryon radius using the corresponding quark wave function. In fact, this calculation shows a slight increase of the inmedium radius. (See Table II in Ref. [21].)

In Figs. 2-4 we show, respectively, the density dependence of the Lorentz-scalar effective baryon masses, inmedium bag radii, and the lowest mode bag eigenvalues. In Figs. 2 and 4, we respectively, show the density dependence of the Lorentz-scalar baryon effective masses and the lowest mode bag eigenvalues, and each panel of the corresponding figures is, for nucleon and strange baryons (top panel), for charm baryons (bottom left panel), and for bottom baryons (bottom right panel).

For the Lorentz-scalar effective masses shown in Fig. 2, one can notice a very interesting feature for the bottom baryons (bottom right panel). The Lorentz-scalar effective mass of $\Sigma_{b}$ becomes smaller than that of $\Xi_{b}$, namely, $m_{\Sigma_{b}}^{*}<$ $m_{\Xi_{b}}^{*}$ at a baryon density range larger than about $0.3 \rho_{0}$, although vacuum masses satisfy $m_{\Sigma_{b}}>m_{\Xi_{b}}$ [55] (see Table II). This is indeed interesting and can be understood as follows. The $\Sigma_{b}$ baryon contains two light quarks, while the $\Xi_{b}$ baryon contains one. Because the light-quark condensates are much more sensitive to the nuclear density change than those of the strange, charm, and bottom quark ones, one can expect the partial restoration of chiral symmetry to take place faster for $\Sigma_{b}$ than $\Xi_{b}$ as the nuclear density increases. Or in the QMC model picture, since the scalar potential is roughly proportional to the number of valence light quarks $[14,25,36]$, the Lorentz-scalar effective

TABLE II. The parameters related with the zero-point energy $z_{B}$; baryon masses and the bag radii in free space [at normal nuclear matter density, $\left.\rho_{0}=0.15 \mathrm{fm}^{-3}\right] m_{B}(\mathrm{MeV}), R_{B}(\mathrm{fm})\left[m_{B}^{*}, R_{B}^{*}\right]$; and the lowest mode bag eigenvalues $x_{1}, x_{2}, x_{3}\left[x_{1}^{*}, x_{2}^{*}, x_{3}^{*}\right]$ of baryon $B\left(q_{1}, q_{2}, q_{3}\right)$ with the corresponding valence quarks $q_{1}, q_{2}, q_{3}$ in the baryon $B$, where $z_{B}$ 's are kept the same as those in vacuum, i.e., density independent. Free space mass values $m_{B}$ for the heavy baryons from Ref. [55], those for the strange hyperons from Ref. [14], and the nucleon bag radius $R_{N}=0.8 \mathrm{fm}$ (and $m_{q}=5 \mathrm{MeV}$ ) are inputs. The light quarks are indicated by $q=u$ or $d$. Note that the baryons containing at least one light quark $q$ are modified in the medium in the QMC model, but $\Omega, \Omega_{c}$, and $\Omega_{b}$ are not modified in the QMC model. We recall that some inputs are updated from those in Refs. [14,15] based on the data [55]. For the recent data for $\Sigma_{b}$, see Ref. [56], which gives the averaged mass of $m_{\Sigma_{b}}=5813.1 \mathrm{MeV}$, to be consistent with the value extracted from Ref. [55].

\begin{tabular}{|c|c|c|c|c|c|c|c|c|c|c|c|}
\hline$\underline{B\left(q_{1}, q_{2}, q_{3}\right)}$ & $z_{B}$ & $m_{B}$ & $R_{B}$ & $x_{1}$ & $x_{2}$ & $x_{3}$ & $m_{B}^{*}$ & $R_{B}^{*}$ & $x_{1}^{*}$ & $x_{2}^{*}$ & $x_{3}^{*}$ \\
\hline$J(q q q)$ & 3.295 & 939.0 & 0.800 & 2.052 & 2.052 & 2.052 & 754.5 & 0.786 & 1.724 & 1.724 & 1.724 \\
\hline$(u d s)$ & 3.131 & 1115.7 & 0.806 & 2.053 & 2.053 & 2.402 & 992.7 & 0.803 & 1.716 & 1.716 & 2.401 \\
\hline$(q q s)$ & 2.810 & 1193.1 & 0.827 & 2.053 & 2.053 & 2.409 & 1070.4 & 0.824 & 1.705 & 1.705 & 2.408 \\
\hline$(q s s)$ & 2.860 & 1318.1 & 0.820 & 2.053 & 2.406 & 2.406 & 1256.7 & 0.818 & 1.708 & 2.406 & 2.406 \\
\hline$(s s s)$ & 1.930 & 1672.5 & 0.869 & 2.422 & 2.422 & 2.422 & ot applicable & ot applicable & not applica & ot applicable & not applicable \\
\hline${ }_{c}(u d c)$ & 42 & 280 & 4 & 2.053 & 2.053 & 2.879 & 216 & 0.851 & 1.6 & 1.691 & \\
\hline$c(q q c)$ & 0.903 & 2453.5 & 0.892 & 2.054 & 2.054 & 2.889 & 2331.8 & 0.889 & 1.671 & 1.671 & \\
\hline$(q s c)$ & 1.445 & 2469.4 & 0.860 & 2.053 & 2.419 & 2.880 & 2408.3 & 0.859 & 1.687 & 2.418 & 2.880 \\
\hline$\Omega_{c}(s s c)$ & 1.057 & 2695.2 & 0.876 & 2.424 & 2.424 & 2.884 & ot applicab & not applicable & ot applicable & ot applicable & not applicable \\
\hline${ }_{b}(u d b)$ & 0.622 & 5619.6 & 0.930 & 2.054 & 2.054 & 3.0 & 0.8 & 0.927 & & & \\
\hline$b(q q b)$ & -1.554 & 5813.4 & 0.968 & 2.054 & 2.054 & 3.066 & 5692.8 & 0.966 & 1.630 & 1.630 & 3.066 \\
\hline$b(q s b)$ & -0.785 & 5793.2 & 0.933 & 2.054 & 2.441 & 3.063 & 5732.7 & 0.931 & 1.649 & 2.440 & 3.063 \\
\hline $2_{b}(s s b)$ & -1.327 & 6046.1 & 0.951 & 2.446 & 2.446 & 3.065 & ot applicable & not applicable & not applicable & not applicable & not applicable \\
\hline
\end{tabular}



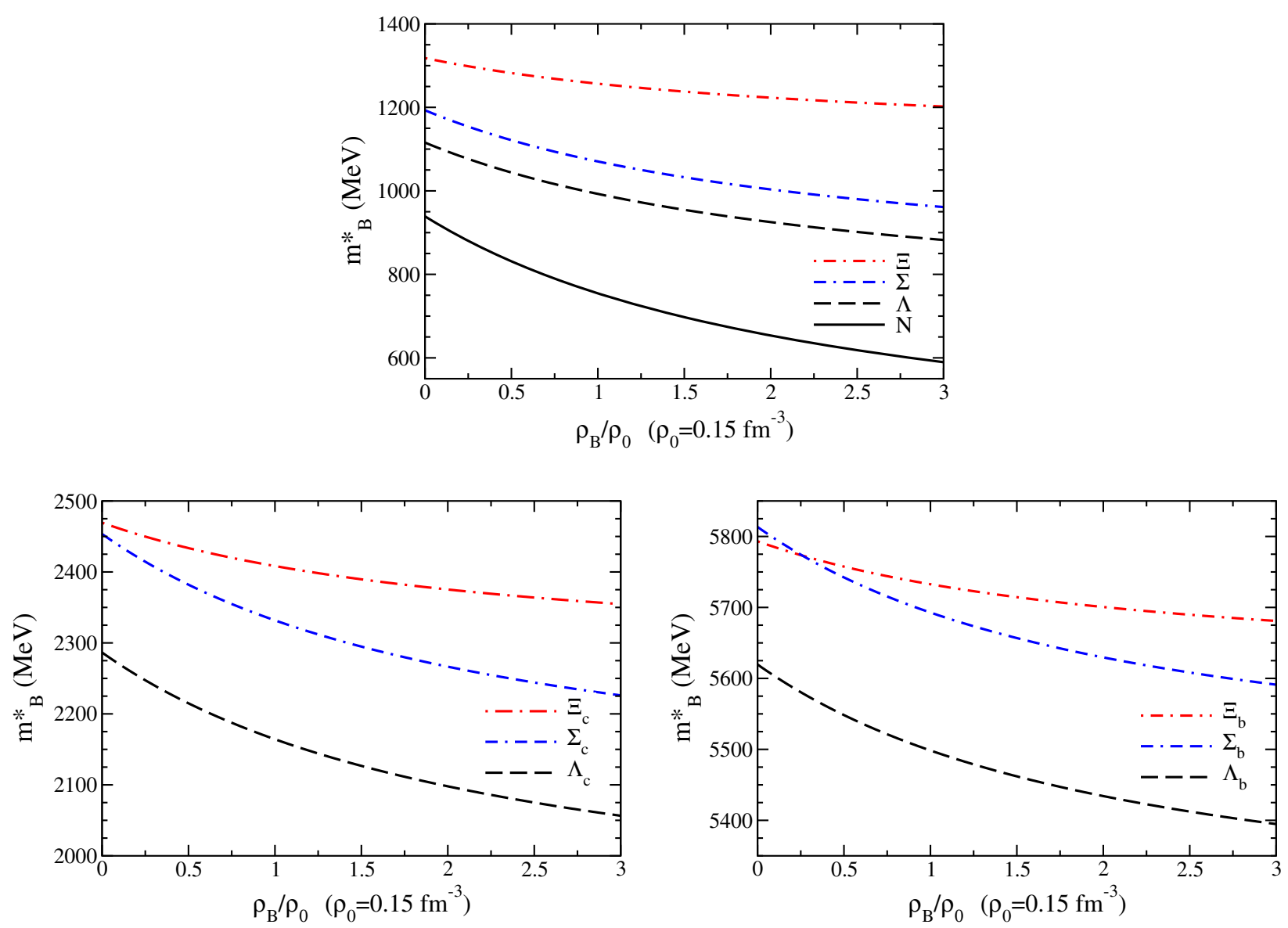

FIG. 2. Density dependence of Lorentz-scalar baryon effective masses in symmetric nuclear matter.

mass of $\Sigma_{b}$ decreases faster than that of $\Xi_{b}$ as the nuclear matter density increases.

The result of the reverse in the Lorentz-scalar effective masses of $\Sigma_{b}$ and $\Xi_{b}$ is one of the main predictions of this article. We must seek how this interesting prediction can possibly be connected with experimental observables. This would give very important information on the dynamical symmetry breaking and the partial restoration of chiral (dynamical) symmetry. However, the story is not that straightforward and simple as discussed above since the baryons (light quarks) also feel repulsive Lorentz-vector potentials in addition to the attractive Lorentz-scalar potentials. Thus, we must take into account the effects of the repulsive vector potentials for considering more realistic/ practical experimental situations, and we will study this later.

Concerning the in-medium bag radii shown in Fig. 3, one can notice that all the in-medium bag radii decrease as the nuclear matter density increases. In particular, the decrease for the nucleon case is the largest.

As for the lowest mode bag eigenvalues shown in Fig. 4, they also decrease as the nuclear matter density increases, particularly for the light quarks, while the heavier quarks have smaller decreases.

In connection with the Lorentz-scalar effective baryon masses shown in Fig. 2, it has been found that the function
$C_{B}(\sigma)\left(B=N, \Lambda, \Sigma, \Xi, \Lambda_{c}, \Sigma_{c}, \Xi_{c}, \Lambda_{b}, \Sigma_{b}, \Xi_{b}\right)$ [see Eq. (13) and above] can be parametrized as a linear form in the $\sigma$ field, $g_{\sigma} \sigma$, for a practical use $[20,21,25]$ :

$$
\begin{aligned}
C_{B}(\sigma)= & 1-a_{B} \times\left(g_{\sigma} \sigma\right), \\
& \left(B=N, \Lambda, \Sigma, \Xi, \Lambda_{c}, \Sigma_{c}, \Xi_{c}, \Lambda_{b}, \Sigma_{b}, \Xi_{b}\right) .
\end{aligned}
$$

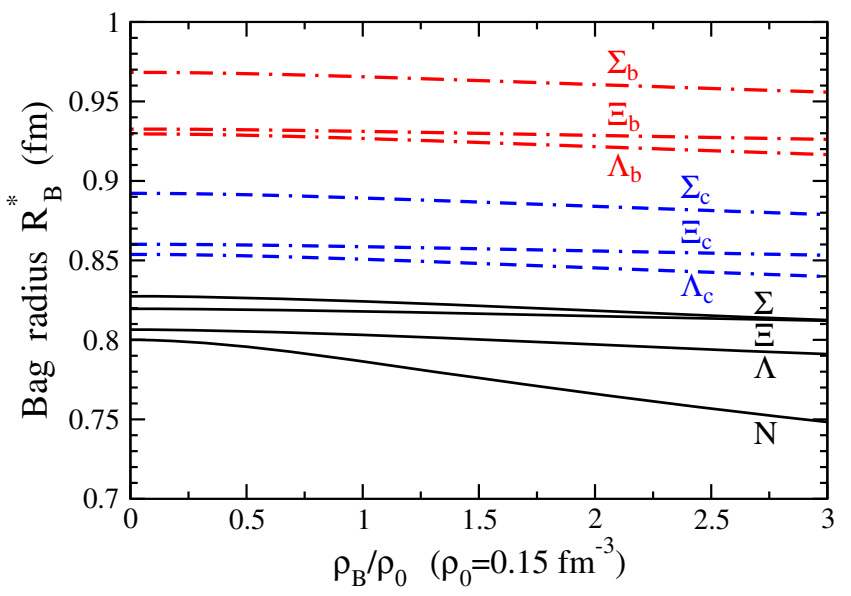

FIG. 3. Density dependence of in-medium bag radii in symmetric nuclear matter. 

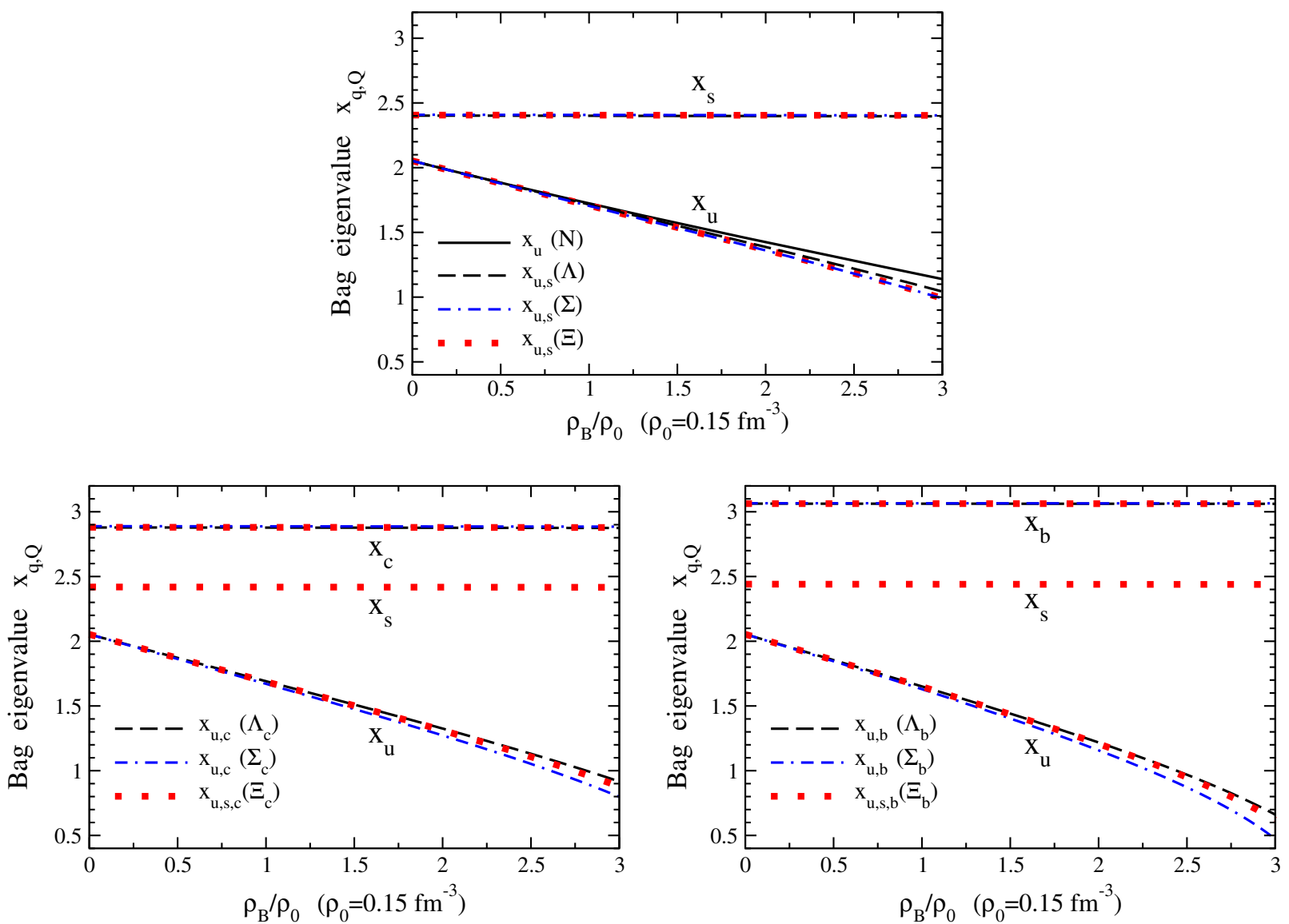

FIG. 4. Density dependence of the lowest mode bag eigenvalues in symmetric nuclear matter.

The values obtained for $a_{B}$ are listed in Table III. This parametrization works very well up to about three times the normal nuclear matter density $3 \rho_{0}$. Then, the effective mass of baryons $B$ in nuclear matter is well approximated by

$$
\begin{aligned}
& m_{B}^{*} \simeq m_{B}-\frac{n_{q}}{3} g_{\sigma}\left[1-\frac{a_{B}}{2}\left(g_{\sigma} \sigma\right)\right] \sigma, \\
&\left(B=N, \Lambda, \Sigma, \Xi, \Lambda_{c}, \Sigma_{c}, \Xi_{c}, \Lambda_{b}, \Sigma_{b}, \Xi_{b}\right),
\end{aligned}
$$

with $n_{q}$ being the valence light-quark number in the baryon $B$. See Eqs. (4) and (5) to compare with $g^{N, Y}(\sigma)$ and the above expression. For the $\Sigma_{b}$ and $\Xi_{b}$ baryons, $n_{q}$ are, respectively,

TABLE III. Slope parameters, $a_{B}\left(B=N, \Lambda, \Sigma, \Xi, \Lambda_{c}, \Sigma_{c}, \Xi_{c}\right.$, $\left.\Lambda_{b}, \Sigma_{b}, \Xi_{b}\right)$. Note that the tiny differences in values of $a_{B}$ from those in Refs. $[14,15]$ are due to the differences in the number of data points for calculating $a_{B}$, but such differences in $a_{B}$ give negligible effects.

\begin{tabular}{cccccc}
\hline \hline$a_{B}$ & $\times 10^{-4} \mathrm{MeV}^{-1}$ & $a_{B}$ & $\times 10^{-4} \mathrm{MeV}^{-1}$ & $a_{B}$ & $\times 10^{-4} \mathrm{MeV}^{-1}$ \\
\hline$a_{N}$ & 9.1 & - & - & - & - \\
$a_{\Lambda}$ & 9.3 & $a_{\Lambda_{c}}$ & 9.9 & $a_{\Lambda_{b}}$ & 10.8 \\
$a_{\Sigma}$ & 9.6 & $a_{\Sigma_{c}}$ & 10.3 & $a_{\Sigma_{b}}$ & 11.2 \\
$a_{\Xi}$ & 9.5 & $a_{\Xi_{c}}$ & 10.0 & $a_{\Xi_{b}}$ & 10.8 \\
\hline \hline
\end{tabular}

two and one in Eq. (27) with $a_{\Sigma_{b}} \simeq a_{\Xi_{b}}$ from Table III. Then, one can confirm that the decrease in the Lorentz-scalar effective mass for $\Sigma_{b}$ is larger than that for $\Xi_{b}$ as the nuclear matter density or the $\sigma$ mean field magnitude increases.

To analyze more carefully the interesting findings for the $\Sigma_{b}$ and $\Xi_{b}$ baryon Lorentz-scalar effective masses, that their magnitudes reverse as, $m_{\Xi_{b}}^{*}<m_{\Sigma_{b}}^{*}$, in medium, we next discuss the "excitation energies" of baryons, to study the total energies (potentials) in a nonrelativistic sense, the Lorentz-scalar plus Lorentz-vector potentials focusing on the $\Sigma_{b}$ and $\Xi_{b}$ baryons. First, results for the attractive scalar and repulsive vector potentials are shown separately in Fig. 5, for nucleon and strange baryons (top panel), charm baryons (bottom left panel), and bottom baryons (bottom right panel). For the repulsive vector potentials, we show here only one case, the one including the effective "Pauli potentials" introduced in Eq. (12), denoted by "vector (+Pauli)." One can see the similarity in the amounts of the scalar and vector(+Pauli) potentials among the corresponding strange, charm, and bottom sector baryons, namely, among those three baryons in each bracket, $\left(\Lambda, \Lambda_{c}, \Lambda_{b}\right)$, $\left(\Sigma, \Sigma_{c}, \Sigma_{b}\right)$, and $\left(\Xi, \Xi_{c}, \Xi_{b}\right)$.

Now we show in Fig. 6 the Lorentz-scalar effective masses and excitation energies (total energies) and the Lorentz-scalar effective masses plus vector potentials for 

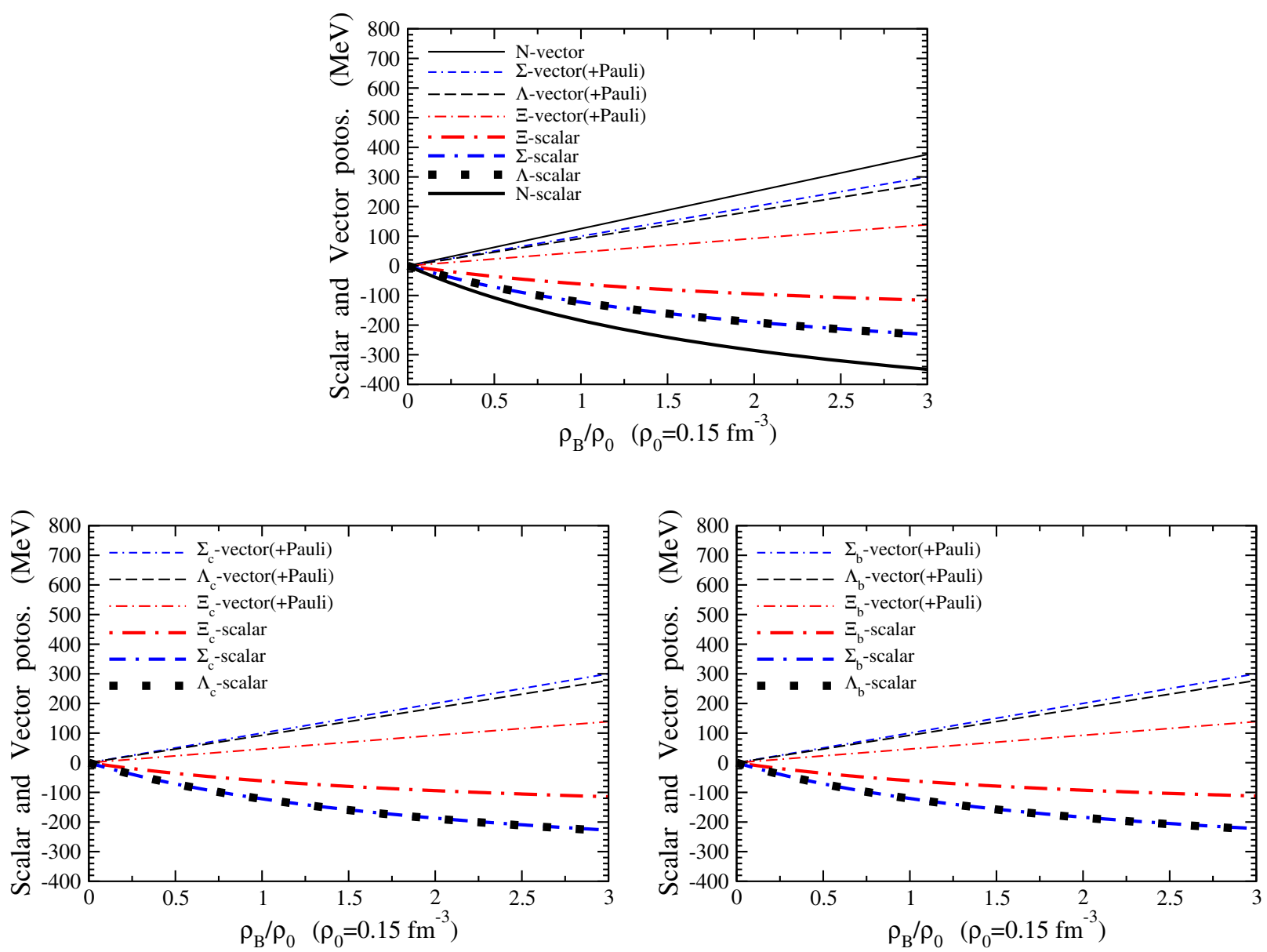

FIG. 5. Attractive Lorentz-scalar and repulsive Lorentz-vector potentials of baryons in symmetric nuclear matter. In figures, "vector +Pauli" denotes that the effective Pauli potentials are added, and "potos." in each vertical axis is the abbreviation for "potentials."

the two cases of the vector potentials focusing on $\Sigma_{b}$ and $\Xi_{b}$. The left panel is the case with the Pauli potentials, while the right panel is the case without the Pauli potentials. Recall that, because the mass difference between the $\Lambda_{b}-\Sigma_{b}$ system is much larger than that for the $\Lambda-\Sigma$ and $\Lambda_{c}-\Sigma_{c}$ systems, it is expected that the effective Pauli potentials should be smaller for the $\Lambda_{b}, \Sigma_{b}$, and $\Xi_{b}$ baryons than the corresponding strange and charm sector baryons. Thus, one can regard the more realistic case when we consider it without the Pauli potentials, as shown in the right panel of Fig. 6 .

We discuss separately the two cases of the vector potentials. First, for the case with the Pauli potentials shown in the left panel of Fig. 6, the excitation energies (total potentials) for the $\Sigma_{b}$ and $\Xi_{b}$ never reverse in magnitudes, and the excitation energy of $\Sigma_{b}$ is always larger than that for $\Xi_{b}$. The smallest excitation energy difference is about a few tens of $\mathrm{MeV}$, and it is larger for $\Sigma_{b}$. For the nuclear matter density larger than around $\rho_{0}$, the difference in the excitation energies increases.

Next, the case without the Pauli potentials, which may be expected to be more realistic, is shown in the right panel of Fig. 6. Interestingly, in the nuclear matter density range $0.5 \rho_{0}<\rho_{B}<1.5 \rho_{0}$, the two excitation energies for $\Sigma_{b}$ and $\Xi_{b}$ are nearly degenerate. This means that $\Sigma_{b}$ and $\Xi_{b}$ can be produced at rest with the nearly same energy costs. This may imply the emergence of many interesting phenomena, for example, in heavy ion reactions and reactions in the systems of a dense nuclear medium, such as in the deep core of a neutron (compact) star.

The results shown in Fig. 6 suggest that the two different types of vector potentials may possibly be distinguished and give important information on the dynamical symmetry breaking and partial restoration of chiral symmetry, by studying the heavy bottom baryon properties in a medium. For proving these suggestions, we have to seek what kind of experiments can be made to get a clue, in particular, for the Lorentz-scalar effective masses of $\Sigma_{b}$ and $\Xi_{b}$. It might be very interesting to measure the valence quark (parton) distributions of $\Sigma_{b}$ and $\Xi_{b}$ in a medium, since the supports of the parton distributions of these baryons reflect their excitation energies. Another possibility may be to measure the strangeness-changing semileptonic weak decay of 

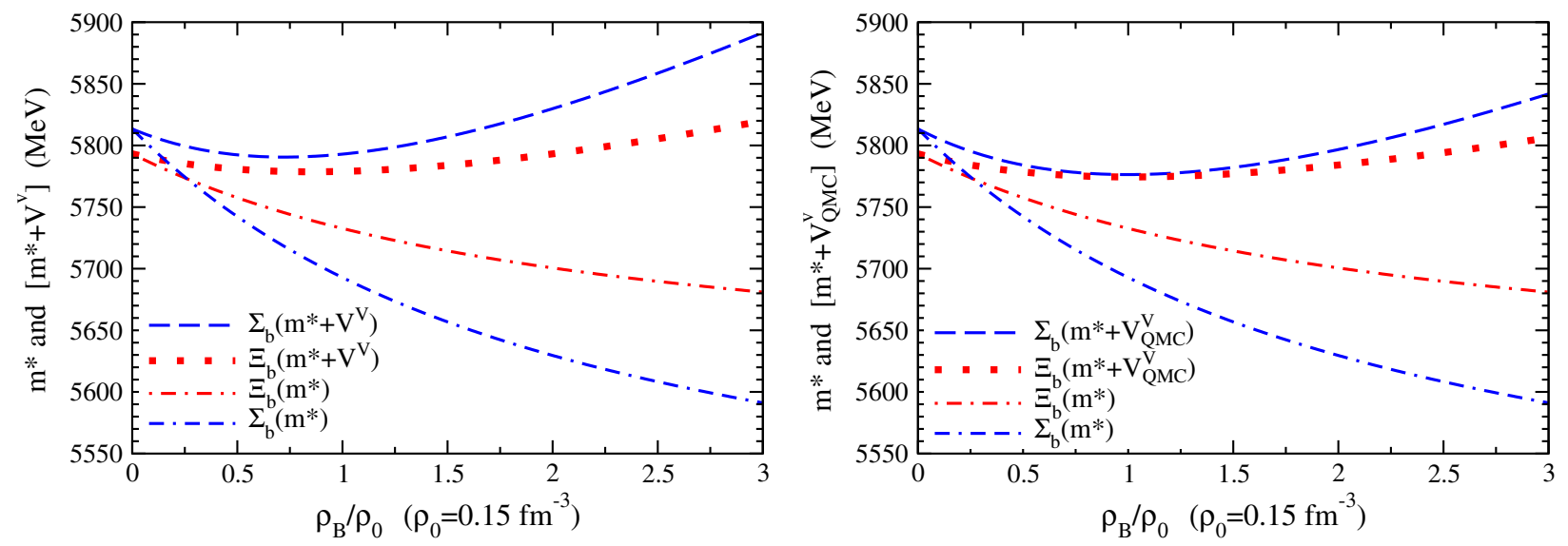

FIG. 6. Effective masses and excitation energies (total energies) of $\Sigma_{b}$ and $\Xi_{b}$ baryons for the two cases of the vector potentials, with (left panel) and without (right panel) the Pauli potentials.

$\Xi_{b} \rightarrow \Sigma_{b}$ in a medium, which again reflects their excitation energy difference in a medium.

\section{SUMMARY AND DISCUSSION}

In this article we have completed the study of baryon properties in symmetric nuclear matter in the quark-meson coupling model, for the low-lying strange, charm, and bottom baryons which contain at least one light quark. We have presented the density dependence of the Lorentzscalar effective masses, bag radii, the lowest mode bag eigenvalues, and vector potentials for the baryons.

We predict that the Lorentz-scalar effective mass of $\Sigma_{b}$ becomes smaller than that of $\Xi_{b}$ in the nuclear matter density range larger than $\simeq 0.3 \rho_{0}\left(\rho_{0}=0.15 \mathrm{fm}^{-3}\right)$, while in vacuum the mass of $\Sigma_{b}$ is larger than that of $\Xi_{b}$. We also give a parametrization for the Lorentz-scalar effective masses of the baryons treated in this article as a function of the scalar mean field for convenient use.

We have further studied the effects of the two different repulsive Lorentz-vector potentials to estimate the excitation (total) energies focusing on $\Sigma_{b}$ and $\Xi_{b}$ baryons. In the case without the effective Pauli potentials, which is expected to be more realistic, the excitation energies for the $\Sigma_{b}$ and $\Xi_{b}$ baryons are predicted to be nearly degenerate in the nuclear matter density range of about $\left[0.3 \rho_{0}, 1.5 \rho_{0}\right]$. Thus, the production of $\Sigma_{b}$ and $\Xi_{b}$ baryons costs nearly the same energy at rest in this nuclear matter density range, and this may imply many interesting phenomena in heavy ion collisions and reactions involving them in the deep core of a neutron (compact) star.

To make possible connections of the findings for the Lorentz-scalar effective masses and/or excitation energies of $\Sigma_{b}$ and $\Xi_{b}$ baryons with experimental observables, we need to seek relevant experimental methods and situations. It might be very interesting to measure the valence quark (parton) distributions of $\Sigma_{b}$ and $\Xi_{b}$ in a medium, since the supports of the parton distributions of these baryons reflect their excitation energies. Another possibility may be to measure the strangeness-changing semileptonic weak decay of $\Xi_{b} \rightarrow \Sigma_{b}$ in a medium, which again reflects their excitation energy difference in a medium.

In conclusion, studies of heavy baryon properties, in particular $\Sigma_{b}$ and $\Xi_{b}$ baryons in a nuclear medium, can provide us with very interesting and important information on the dynamical symmetry breaking and partial restoration of chiral symmetry, as well as the roles of the light quarks in a medium.

\section{ACKNOWLEDGMENTS}

This work was partially supported by the Conselho Nacional de Desenvolvimento Científico e Tecnológico (CNPq) Process No. 400826/2014-3 and Process No. 308088/2015-8, and was also part of the projects, Instituto Nacional de Ciência e Tecnologia-Nuclear Physics and Applications (INCT-FNA), Brazil, Process No. 464898/2014-5, and FAPESP Temático, Brazil, Process No. 2017/05660-0. 
[1] E. E. Jenkins and A. V. Manohar, Phys. Lett. B 255, 558 (1991).

[2] M. B. Wise, Phys. Rev. D 45, R2188 (1992).

[3] For a review, see J. G. Korner, M. Kramer, and D. Pirjol, Prog. Part. Nucl. Phys. 33, 787 (1994).

[4] C. Albertus, J. E. Amaro, E. Hernandez, and J. Nieves, Nucl. Phys. A740, 333 (2004).

[5] X. Liu, H. X. Chen, Y. R. Liu, A. Hosaka, and S. L. Zhu, Phys. Rev. D 77, 014031 (2008).

[6] E. E. Jenkins, Phys. Rev. D 77, 034012 (2008).

[7] Z. S. Brown, W. Detmold, S. Meinel, and K. Orginos, Phys. Rev. D 90, 094507 (2014).

[8] V. Simonis, arXiv:1803.01809.

[9] N. Isgur and M. B. Wise, Phys. Rev. Lett. 66, 1130 (1991).

[10] T. Mannel, W. Roberts, and Z. Ryzak, Nucl. Phys. B355, 38 (1991).

[11] For a review, see M. Neubert, Phys. Rep. 245, 259 (1994).

[12] G. P. Lepage, L. Magnea, C. Nakhleh, U. Magnea, and K. Hornbostel, Phys. Rev. D 46, 4052 (1992).

[13] N. Brambilla, A. Pineda, J. Soto, and A. Vairo, Nucl. Phys. B566, 275 (2000).

[14] For a review, see K. Saito, K. Tsushima, and A. W. Thomas, Prog. Part. Nucl. Phys. 58, 1 (2007).

[15] For a review, see G. Krein, A. W. Thomas, and K. Tsushima, Prog. Part. Nucl. Phys. 100, 161 (2018).

[16] For a review, see A. Hosaka, T. Hyodo, K. Sudoh, Y. Yamaguchi, and S. Yasui, Prog. Part. Nucl. Phys. 96, 88 (2017).

[17] K. Azizi, N. Er, and H. Sundu, Nucl. Phys. A960, 147 (2017); A962, 122(E) (2017).

[18] K. Azizi and N. Er, Nucl. Phys. A970, 422 (2018).

[19] P. A. M. Guichon, Phys. Lett. B 200, 235 (1988).

[20] P. A. M. Guichon, K. Saito, E. N. Rodionov, and A. W. Thomas, Nucl. Phys. A601, 349 (1996).

[21] K. Saito, K. Tsushima, and A. W. Thomas, Nucl. Phys. A609, 339 (1996).

[22] K. Saito, K. Tsushima, and A. W. Thomas, Phys. Rev. C 55, 2637 (1997).

[23] J. R. Stone, P. A. M. Guichon, P. G. Reinhard, and A. W. Thomas, Phys. Rev. Lett. 116, 092501 (2016).

[24] P. A. M. Guichon, J. R. Stone, and A. W. Thomas, Prog. Part. Nucl. Phys. 100, 262 (2018).

[25] K. Tsushima, K. Saito, J. Haidenbauer, and A. W. Thomas, Nucl. Phys. A630, 691 (1998).

[26] K. Tsushima, K. Saito, and A. W. Thomas, Phys. Lett. B 411, 9 (1997); 421, 413 (1998).

[27] P. A. M. Guichon, A. W. Thomas, and K. Tsushima, Nucl. Phys. A814, 66 (2008).

[28] K. Tsushima and F. C. Khanna, Phys. Rev. C 67, 015211 (2003).

[29] K. Tsushima and F. C. Khanna, Prog. Theor. Phys. Suppl. 149, 160 (2003).

[30] K. Tsushima and F. C. Khanna, J. Phys. G 30, 1765 (2004).
[31] K. Saito, K. Tsushima, and A. W. Thomas, Phys. Rev. C 56, 566 (1997).

[32] K. Tsushima, K. Saito, A. W. Thomas, and S. V. Wright, Phys. Lett. B 429, 239 (1998); 436, 453(E) (1998).

[33] K. Tsushima, D. H. Lu, A. W. Thomas, and K. Saito, Phys. Lett. B 443, 26 (1998).

[34] K. Tsushima, D. H. Lu, A. W. Thomas, K. Saito, and R. H. Landau, Phys. Rev. C 59, 2824 (1999).

[35] A. Sibirtsev, K. Tsushima, K. Saito, and A. W. Thomas, Phys. Lett. B 484, 23 (2000).

[36] K. Tsushima and F. C. Khanna, Phys. Lett. B 552, 138 (2003).

[37] A. Sibirtsev, K. Tsushima, and A. W. Thomas, Eur. Phys. J. A 6, 351 (1999).

[38] R. Shyam, K. Tsushima, and A. W. Thomas, Phys. Lett. B 676, 51 (2009).

[39] K. Tsushima, P. A. M. Guichon, R. Shyam, and A. W. Thomas, Int. J. Mod. Phys. E 19, 2546 (2010).

[40] R. Shyam, K. Tsushima, and A. W. Thomas, Nucl. Phys. A881, 255 (2012).

[41] R. Chatterjee, R. Shyam, K. Tsushima, and A. W. Thomas, Nucl. Phys. A913, 116 (2013).

[42] K. Tsushima, R. Shyam, and A. W. Thomas, Few-Body Syst. 54, 1271 (2013).

[43] R. Shyam and K. Tsushima, Phys. Rev. D 94, 074041 (2016).

[44] R. Shyam and K. Tsushima, Phys. Lett. B 770, 236 (2017).

[45] R. Shyam and K. Tsushima, Few-Body Syst. 59, 18 (2018).

[46] D. L. Whittenbury, J. D. Carroll, A. W. Thomas, K. Tsushima, and J. R. Stone, Phys. Rev. C 89, 065801 (2014).

[47] A. W. Thomas, D. L. Whittenbury, J. D. Carroll, K. Tsushima, and J. R. Stone, Eur. Phys. J. Web Conf. 63, 03004 (2013).

[48] K. Tsushima, T. Maruyama, and A. Faessler, Nucl. Phys. A535, 497 (1991).

[49] T. Maruyama, K. Tsushima, and A. Faessler, Nucl. Phys. A537, 303 (1992).

[50] G. Krein, A. W. Thomas, and K. Tsushima, Nucl. Phys. A650, 313 (1999).

[51] J. D. Walecka, Ann. Phys. (N.Y.) 83, 491 (1974).

[52] B. D. Serot and J.D. Walecka, in The relativistic nuclear many-body problem, edited by J. W. Negele and E. Vogt, Adv. in Nuclear Physics Vol. 16(1) (Plenum Press, New York, 1986).

[53] P. A. M. Guichon, H. H. Matevosyan, N. Sandulescu, and A. W. Thomas, Nucl. Phys. A772, 1 (2006).

[54] M. Dutra, O. Lourenco, J. S. S. Martins, A. Delfino, J. R. Stone, and P. D. Stevenson, Phys. Rev. C 85, 035201 (2012).

[55] C. Patrignani et al. (Particle Data Group), Chin. Phys. C 40, 100001 (2016) and 2017 update.

[56] R. Aaij et al. (LHCb Collaboration), arXiv:1809.07752. 